\title{
A new resistance function for two rigid spheres in a uniform compressible low-Reynolds-number flow
}

\author{
Aditya S. Khair, Manuj Swaroop, and John F. Brady \\ Division of Chemistry and Chemical Engineering, California Institute of Technology, Pasadena, \\ California 91125
}

(Received 13 October 2005; accepted 10 March 2006; published online 21 April 2006)

\begin{abstract}
The pressure moment of a rigid particle is defined as the trace of the first moment of the surface stress acting on the particle. We calculate the pressure moments of two unequal rigid spheres immersed in a uniform compressible linear flow, using twin multipole expansions and lubrication theory. Following the practice established in previous studies on two-body hydrodynamic interactions at low Reynolds number, the results are expressed in terms of a new (stresslet) resistance function. (C) 2006 American Institute of Physics. [DOI: 10.1063/1.2194559]
\end{abstract}

\section{INTRODUCTION}

In low-Reynolds-number fluid dynamics, it is common to characterize interactions between particles using the moments of the surface stress acting on each particle. Traditionally, the first moment has been split into two parts: an antisymmetric part, which gives the torque acting on a particle, and a traceless symmetric part called the stresslet. More recently, Jeffrey, Morris, and Brady ${ }^{1}$ (hereafter JMB) calculated the trace of the first moment, which they termed the "pressure moment," and used it to determine the osmotic pressure in a dilute colloidal dispersion. Specifically, they computed the pressure moments for two unequal rigid spheres immersed in an incompressible linear flow, i.e., a flow with a traceless ambient rate of strain tensor. However, just as the pressure moment is the trace of the first moment of the surface stress, it is natural to consider the contribution to the pressure moment from compressible linear flows (for which the trace of the rate of strain tensor is nonzero). Put another way, if one allows the stresslet to possess a trace (i.e., the pressure moment), one must consider the contribution to it from a rate of strain tensor with nonzero trace. ${ }^{2}$

One should not think, however, that the present exercise is of purely mathematical interest. Recently, Brady, Khair, and Swaroop ${ }^{3}$ (hereafter BKS) computed the bulk viscosity (also known as the second or expansion viscosity) of a colloidal dispersion to second order in volume fraction of the (rigid spherical) suspended particles. This calculation involves determination of the dispersion microstructure, which reflects a balance between an imposed uniform expansion flow [for which the trace of the rate of strain tensor is a (nonzero) constant], serving to drive the dispersion out of equilibrium, and Brownian diffusion of the colloidal particles, which acts to restore equilibrium. There is a contribution to the bulk viscosity due to the inability of the (rigid) particles to deform in the expansion flow, which is reflected mathematically as an average of the pressure moments of the particles weighted over the nonequilibrium microstructure. Thus, the calculation of the pressure moments for two rigid spheres immersed in a uniform compressible flow is indeed of practical interest. Furthermore, we anticipate that the re- sults presented in this work may be useful in future studies of colloidal dispersions in compressible flow fields.

Finally, the function relating the pressure moment to the trace of the rate of strain "completes" the set of two-body hydrodynamic resistance functions for spherical particles.

We proceed by recalling the constitutive equation for the stress tensor $\boldsymbol{\sigma}$ in a compressible Newtonian fluid (see Secs. 3.3 and 3.4 of Batchelor ${ }^{4}$ )

$$
\boldsymbol{\sigma}=-p_{t h} \boldsymbol{I}+2 \eta \boldsymbol{e}+\left(\kappa-\frac{2}{3} \eta\right)(\boldsymbol{\nabla} \cdot \boldsymbol{u}) \boldsymbol{I},
$$

where $p_{t h}$ is the thermodynamic pressure, ${ }^{5} \boldsymbol{e}=\frac{1}{2}\left[\nabla \boldsymbol{u}+(\boldsymbol{\nabla} \boldsymbol{u})^{\dagger}\right]$ is the rate of strain tensor with $\boldsymbol{u}$ the fluid velocity, $\eta$ is the shear viscosity, $\kappa$ is the bulk viscosity, and $\boldsymbol{I}$ is the identity tensor. The pressure moment $S$ of a particle is given by

$$
S=-\int\left[\boldsymbol{x}^{\prime} \cdot \boldsymbol{\sigma} \cdot \boldsymbol{n}-3 \kappa \boldsymbol{n} \cdot \boldsymbol{u}\right] d A ;
$$

the integral is over the surface of the particle with normal $\boldsymbol{n}$ pointing into the fluid, and $\boldsymbol{x}^{\prime}$ is the position vector measured from the center of the particle. For rigid particles, the normal component of the fluid velocity vanishes on the particle surface; thus (1) reduces to

$$
S=-\int \boldsymbol{x}^{\prime} \cdot \boldsymbol{\sigma} \cdot \boldsymbol{n} d A .
$$

In Stokes flow the velocity field can be decomposed into a uniform rate of expansion everywhere in the fluid (which, by definition, has an isotropic rate of strain tensor) and a disturbance flow created by any immersed particles (and their fluid-mediated interactions), which satisfies the usual incompressible Stokes equations; that is,

$$
\boldsymbol{u}=\frac{1}{3} E \boldsymbol{x}+\boldsymbol{u}^{s},
$$

with $\boldsymbol{\nabla} \cdot \boldsymbol{u}=E$ and $\boldsymbol{\nabla} \cdot \boldsymbol{u}^{s}=0$. In turn, the fluid stress can be decomposed into a(n) (isotropic) contribution due to the uniform expansion flow, $\boldsymbol{\sigma}^{E}=-\left(p_{t h}-\kappa E\right) \boldsymbol{I}$, and a disturbance stress $\boldsymbol{\sigma}^{s}=-p^{s} \boldsymbol{I}+2 \eta \boldsymbol{e}^{s}$, with $\boldsymbol{\nabla} \cdot \boldsymbol{\sigma}^{s}=0$. Here, $p^{s}$ is the dynamical pressure of the incompressible Stokes flow. Substituting 
this decomposition into (2) we have for the pressure moment

$$
S=\left(p_{t h}-\kappa E\right) \int \boldsymbol{x}^{\prime} \cdot \boldsymbol{n} d A-\int \boldsymbol{x}^{\prime} \cdot \boldsymbol{\sigma}^{s} \cdot \boldsymbol{n} d A,
$$

where the first term on the right-hand side of (3), to be denoted as $S^{c}$, is the contribution to the pressure moment from the uniform expansion flow, and the second term, to be denoted as $S^{S}$, is the contribution from the incompressible (Stokes) disturbance flow. ${ }^{6}$

Now, suppose we have two particles in such a uniform expansion flow. The disturbance stress on the surface of a particle may be written as $\boldsymbol{\sigma}^{s}=\boldsymbol{\sigma}^{s, 0}+\boldsymbol{\sigma}^{s, i}$, i.e., a sum of the single (isolated) particle contribution $\boldsymbol{\sigma}^{s, 0}$ and an "interaction" contribution $\boldsymbol{\sigma}^{s, i}$ due to the presence of the second particle. For a single spherical particle of radius $a$ in the expansion flow, the disturbance velocity is (BKS)

$$
\boldsymbol{u}^{s, 0}=-\frac{1}{3} E a^{3} \boldsymbol{x}^{\prime} / r^{3} \quad \text { and } \quad p^{s, 0}=0,
$$

where $r=\left|\boldsymbol{x}^{\prime}\right|$. [Note, for the single spherical particle $S^{c}$ $=4 \pi a^{3}\left(p_{t h}-\kappa E\right)$.] The disturbance stress is then

$$
\boldsymbol{\sigma}^{s, 0}=-\frac{2}{3} a^{3} \eta E\left(\frac{\boldsymbol{I}}{r^{3}}-\frac{3 \boldsymbol{x}^{\prime} \boldsymbol{x}^{\prime}}{r^{5}}\right) .
$$

Therefore, the disturbance flow contribution to the pressure moment may be written as $S^{s}=S^{s, 0}+S^{s, i}$, where

$$
S^{s, 0}=-\frac{16}{3} \pi a^{3} \eta E
$$

is the single particle ("self") contribution and

$$
S^{s, i}=-\int \boldsymbol{x}^{\prime} \cdot \boldsymbol{\sigma}^{s, i} \cdot \boldsymbol{n} d A
$$

is the contribution due to hydrodynamic interactions with the second particle. Note, (4) is identical to the pressure moment for two particles in an incompressible linear flow, as considered in JMB, for which there is no self-contribution analogous to $S^{s, 0}$. Henceforth, for clarity of presentation, we drop the superscripts $s$ and $i$; they are to be understood.

It is the aim of this work to calculate the (disturbance flow contribution to the) pressure moments for two spherical particles immersed in a uniform expansion flow. As per previous investigations on two-particle hydrodynamic interactions (Jeffrey and Onishi, ${ }^{7}$ Jeffrey, ${ }^{8}$ and JMB), twin multipole expansions and lubrication theory are employed. We follow the nomenclature of these earlier works throughout, and, in the interest of brevity, we do not repeat their derivations and equations here.

\section{RESISTANCE FUNCTIONS}

Consider two rigid spherical particles of radii $a_{1}$ and $a_{2}$, respectively. The pressure moment of sphere $\alpha\left(S_{\alpha}\right)$ is related to the translational velocities $\left(\boldsymbol{U}_{\alpha}\right)$ of the spheres and their rates of strain $\left(\boldsymbol{E}_{\alpha}\right)$ via a generalized matrix equation,

$$
\left(\begin{array}{l}
S_{1} \\
S_{2}
\end{array}\right)=\eta\left(\begin{array}{llll}
\boldsymbol{P}_{11} & \boldsymbol{P}_{12} & \boldsymbol{Q}_{11} & \boldsymbol{Q}_{12} \\
\boldsymbol{P}_{21} & \boldsymbol{P}_{22} & \boldsymbol{Q}_{21} & \boldsymbol{Q}_{22}
\end{array}\right) \cdot\left(\begin{array}{c}
\boldsymbol{U}_{1}-\boldsymbol{U}\left(\boldsymbol{x}_{1}\right) \\
\boldsymbol{U}_{2}-\boldsymbol{U}\left(\boldsymbol{x}_{2}\right) \\
\boldsymbol{E}_{1}-\boldsymbol{E}_{\infty} \\
\boldsymbol{E}_{2}-\boldsymbol{E}_{\infty}
\end{array}\right),
$$

where the ambient velocity field at a point $\boldsymbol{x}$ is given by

$$
\boldsymbol{U}(\boldsymbol{x})=\boldsymbol{U}_{\infty}+\boldsymbol{\Omega}_{\infty} \times \boldsymbol{x}+\boldsymbol{E}_{\infty} \cdot \boldsymbol{x},
$$

with constant $\boldsymbol{U}_{\infty}, \boldsymbol{\Omega}_{\infty}$, and $\boldsymbol{E}_{\infty}$. [Note that the pressure moments in (5) are for the incompressible Stokes disturbance flow only; the contribution from the uniform expansion, $S^{c}$, is not included.] The vectors $\boldsymbol{P}_{\alpha \beta}$ relate the pressure moment to the translational velocities of the particles and are given by

$$
\boldsymbol{P}_{\alpha \beta}=\pi\left(a_{\alpha}+a_{\beta}\right)^{2} X_{\alpha \beta}^{P} \boldsymbol{d},
$$

where $\boldsymbol{d}=\left(\boldsymbol{x}_{2}-\boldsymbol{x}_{1}\right) /\left|\boldsymbol{x}_{2}-\boldsymbol{x}_{1}\right|$ is the dimensionless unit vector directed from particle 1 to particle 2 . The resistance functions $X_{\alpha \beta}^{P}$ have been calculated by JMB. The second-rank tensors $\boldsymbol{Q}_{\alpha \beta}$ relate the pressure moment to the rate of strain, and JMB write $\boldsymbol{Q}_{\alpha \beta}=\pi\left(a_{\alpha}+a_{\beta}\right)^{3} X_{\alpha \beta}^{Q}\left(\boldsymbol{d} \boldsymbol{d}-\frac{1}{3} \boldsymbol{I}\right)$, so that, as with the other resistance tensors contracted with the rate of strain, the $\boldsymbol{Q}_{\alpha \beta}$ are traceless. This is, however, only valid for an incompressible linear flow (for which the rate of strain is traceless); for a (spatially uniform) compressible linear flow there is an additional contribution to the $\boldsymbol{Q}_{\alpha \beta}$ arising from the trace of the rate of strain tensor, with a new resistance function that we designate as $T_{\alpha \beta}^{Q}$. The complete $Q$ function is then

$$
\boldsymbol{Q}_{\alpha \beta}=\pi\left(a_{\alpha}+a_{\beta}\right)^{3}\left[X_{\alpha \beta}^{Q}\left(\boldsymbol{d} \boldsymbol{d}-\frac{1}{3} \boldsymbol{I}\right)+T_{\alpha \beta}^{Q} \frac{1}{3} \boldsymbol{I}\right] ;
$$

hence, by construction, $Q_{i i}^{(\alpha \beta)}=\pi\left(a_{\alpha}+a_{\beta}\right)^{3} T_{\alpha \beta}^{Q}$. The $T_{\alpha \beta}^{Q}$ are functions of $s=2 r /\left(a_{1}+a_{2}\right)$ (with $\left.r=\left|\boldsymbol{x}_{2}-\boldsymbol{x}_{1}\right|\right)$ and $\lambda=a_{2} / a_{1}$ only. The symmetries obeyed by the functions are labeling ones,

$$
T_{\alpha \beta}^{Q}(s, \lambda)=T_{3-\alpha, 3-\beta}^{Q}\left(s, \lambda^{-1}\right) .
$$

\section{THE FUNCTIONS $T_{\alpha \beta}^{Q}$}

\section{A. Method of reflections}

We start by deriving the first couple of terms via the method of reflections. Let sphere 1 deform uniformly at a rate $\boldsymbol{E}_{1}=\frac{1}{3} E_{1} \boldsymbol{I}$. There is no pressure field created by this deformation; however, there is a velocity field $\boldsymbol{u}$ $=1 / 3 E_{1} a_{1}\left(a_{1} / r\right)^{2} \boldsymbol{d}$. Sphere 2 responds to this ambient velocity field by exerting a force on the fluid, which leads to an induced $S_{1}$ of

$$
S_{1}=\frac{8}{3} \pi a_{1}^{3} \eta E_{1}\left(T_{11}^{Q}-2\right)=-\eta \pi\left(a_{1}+a_{2}\right)^{2} X_{12}^{P} \frac{1}{3}\left(a_{1}^{3} / r^{2}\right) E_{1},
$$

implying

$$
T_{11}^{Q}=2+\frac{12 \lambda}{(1+\lambda)^{4} s^{4}}+\cdots .
$$




\section{B. Twin multipole expansions}

Consider sphere 1 deforming at a rate $\boldsymbol{E}_{1}=\frac{1}{3} E_{1} \boldsymbol{I}$ and sphere 2 deforming at $\boldsymbol{E}_{2}=\frac{1}{3} E_{2} \boldsymbol{I}$. We follow Jeffrey and Onishi $^{7}$ (hereafter JO) in equating the surface velocities of the spheres, i.e., we set $a_{1} E_{1}=a_{2} E_{2}$. The functions $\chi, \psi$, and $\omega$ (defined in JO equation 2.6) take the values

$$
\chi_{m n}^{(\alpha)}=-\frac{1}{2} \psi_{m n}^{(\alpha)}=\frac{1}{3} a_{\alpha} E_{\alpha} \delta_{0 m} \delta_{0 n}, \quad \omega_{m n}^{(\alpha)}=0 .
$$

As in JO, defining the problem this way ensures that the coefficients are the same for each sphere, allowing one to drop the label $\alpha$. We expand $p_{0 n}$ and $v_{0 n}$ as a double series in $t_{\alpha}=a_{\alpha} / r$, viz.,

$$
\begin{aligned}
& p_{0 n}=\frac{2}{3} a_{\alpha} E_{\alpha} \sum_{p=0}^{\infty} \sum_{q=0}^{\infty} P_{n p q} t_{\alpha}^{p} t_{3-\alpha}^{q}, \\
& v_{0 n}=\frac{1}{3} a_{\alpha} E_{\alpha} \sum_{p=0}^{\infty} \sum_{q=0}^{\infty} \frac{1}{2 n+1} V_{n p q} t_{\alpha}^{p} t_{3-\alpha}^{q} .
\end{aligned}
$$

The recurrence relations for this problem are

$$
\begin{aligned}
V_{0 p q}= & -\delta_{0 p} \delta_{0 q}, \quad P_{0 p q}=0, \\
V_{n p q}= & P_{n p q}-\frac{2 n}{(2 n+3)(n+1)} \sum_{s=1}^{q}\left(\begin{array}{c}
n+s \\
n
\end{array}\right) P_{s(q-s)(p-n-1)}, \\
P_{n p q}= & \sum_{s=0}^{q} \frac{1}{n+1}\left(\begin{array}{c}
n+s \\
n
\end{array}\right) \\
& \times\left[\frac{n(2 n+1)(2 n s+2-n-s)}{2(2 s-1)(n+s)} P_{s(q-s)(p-n+1)}\right. \\
& -\frac{n(2 n+1)(2 n-1)}{2(2 s+1)} V_{s(q-2-s)(p-n+1)} \\
& \left.-\frac{n(2 n-1)}{2(n+1)} P_{s(q-s)(p-n-1)}\right] .
\end{aligned}
$$

The pressure moment of sphere 1 is

$$
S_{1}=\frac{8}{3} \pi a_{1}^{3} \eta E_{1}\left(T_{11}^{Q}+\frac{(1+\lambda)^{3}}{8 \lambda} T_{12}^{Q}\right),
$$

and from this we find

$$
T_{11}^{Q}+\frac{(1+\lambda)^{3}}{8 \lambda} T_{12}^{Q}=\sum_{n=1}^{\infty} \sum_{p=0}^{\infty} \sum_{q=0}^{\infty} P_{n p q} q_{2}^{n+p} t_{1}^{q+1} .
$$

We define a complimentary problem with $a_{1} E_{1}=-a_{2} E_{2}$, which gives another expression for the pressure moment of sphere 1 ,

$$
T_{11}^{Q}-\frac{(1+\lambda)^{3}}{8 \lambda} T_{12}^{Q}=\sum_{n=1}^{\infty} \sum_{p=0}^{\infty} \sum_{q=0}^{\infty}(-1)^{n+p+q+2} P_{n p q} t_{2}^{n+p} t_{1}^{q+1} .
$$

Thus, we conclude

$$
\begin{aligned}
T_{11}^{Q} & =\sum_{\substack{m=0 \\
m \text { even }}}^{\infty} \sum_{q=1}^{m-1} \sum_{n=1}^{(q+1) / 2} P_{n(q-n)(m-q-1)} \frac{2^{m}}{(1+\lambda)^{m} s^{m}} \lambda^{q} \\
& =\sum_{\substack{m=0 \\
m \text { even }}}^{\infty} \frac{f_{m}(\lambda)}{(1+\lambda)^{m} s^{m}}
\end{aligned}
$$

and

$$
T_{12}^{Q}=\frac{8}{(1+\lambda)^{3}} \sum_{\substack{m=1 \\ m \text { odd }}}^{\infty} \frac{f_{m}(\lambda)}{(1+\lambda)^{m} s^{m}} .
$$

The first few terms of the $f_{m}(\lambda)$ are

$$
\begin{aligned}
& f_{0}=2, f_{1}=f_{2}=f_{3}=0, f_{4}=12 \lambda, \\
& f_{5}=36 \lambda^{4}, f_{6}=108 \lambda^{2}+320 \lambda^{3}, \\
& f_{7}=432 \lambda^{4}+324 \lambda^{5}+432 \lambda^{6}, \\
& f_{8}=432 \lambda^{2}+972 \lambda^{3}+2592 \lambda^{4}+3360 \lambda^{5} .
\end{aligned}
$$

Strictly speaking, through the twin-multipole expansion we calculate the interaction contribution $\left(S^{s, i}\right)$ to the disturbance pressure moment; however, the self-contribution $\left(S^{s, 0}\right)$ is included in the definition of $T_{11}^{Q}$ by requiring $f_{0}=2$. For the complete pressure moment [see (3)] one must, of course, add the contribution due to the uniform expansion flow $\left(S^{c}\right)$.

\section{Lubrication theory}

Let sphere 1 deform (with its center fixed) at a rate $\boldsymbol{E}_{1}$ $=1 / 3 E_{1} \boldsymbol{I}$ in close proximity to sphere 2 (which is at rest). Following Jeffrey, ${ }^{9}$ we write the pressure moment of sphere 1 as

$$
\begin{aligned}
S_{1} & =-\int \boldsymbol{x}^{\prime} \cdot \boldsymbol{\sigma} \cdot \boldsymbol{n} d A \\
& =a_{1} \boldsymbol{F}_{\mathbf{1}} \cdot \boldsymbol{d}-a_{1} \int(\boldsymbol{n}-\boldsymbol{d}) \cdot \boldsymbol{\sigma} \cdot \boldsymbol{n} d A .
\end{aligned}
$$

Substituting the known result for $\boldsymbol{F}_{1}$, we obtain

$$
T_{11}^{Q}=\frac{1}{2} X_{11}^{P}-\frac{3}{8 \pi a_{1}^{2} \eta E_{1}} \int(\boldsymbol{n}-\boldsymbol{d}) \cdot \boldsymbol{\sigma} \cdot \boldsymbol{n} d A .
$$

Performing the integration above (see the Appendix) yields

$$
T_{11}^{Q}=g_{1} \xi^{-1}+g_{2} \ln \xi^{-1}+Q_{11}^{T}+g_{3} \xi \ln \xi^{-1} .
$$

In a similar vein, consideration of the pressure moment for sphere 2 gives

$\frac{1}{8}(1+\lambda)^{3} T_{12}^{Q}=g_{4} \xi^{-1}+g_{5} \ln \xi^{-1}+\frac{1}{8}(1+\lambda)^{3} Q_{12}^{T}+g_{6} \xi \ln \xi^{-1}$,

where

$$
g_{1}=\frac{3 \lambda^{2}}{2(1+\lambda)^{3}}, \quad g_{2}=\frac{3\left(\lambda-13 \lambda^{2}-9 \lambda^{3}\right)}{20(1+\lambda)^{3}},
$$




$$
\begin{aligned}
& g_{3}=\frac{5-274 \lambda-201 \lambda^{2}-190 \lambda^{3}-\frac{1269}{2} \lambda^{4}+\lambda^{5}}{280(1+\lambda)^{3}}, \\
& g_{4}=\frac{3 \lambda^{3}}{2(1+\lambda)^{3}}, \quad g_{5}=\frac{3\left(-4 \lambda^{2}-13 \lambda^{3}-4 \lambda^{4}\right)}{20(1+\lambda)^{3}}, \\
& g_{6}=\frac{1-43 \lambda-92 \lambda^{2}+\frac{123}{2} \lambda^{3}-92 \lambda^{4}-569 \lambda^{5}}{280(1+\lambda)^{3}} .
\end{aligned}
$$

The nondimensional gap width is $\xi=s-2$, and the $Q_{\alpha \beta}^{T}(\lambda)$ are functions that we tabulate here. Note, in the special case of equal spheres, $\lambda=1$, the singular terms in $T_{11}^{Q}$ are the same as those in $T_{12}^{Q}$, but we see that in general this is not true.

\section{ARBITRARY SEPARATIONS}

The singularities cause slow convergence of the series (6) and (7) when $s$ is near 2; following Jeffrey ${ }^{8}$, we remove them by writing

$$
\begin{aligned}
T_{11}^{Q}= & g_{1} \frac{4}{s^{2}-4}+\left[g_{2}+g_{3}\left(\frac{1}{4} s^{2}-1\right)\right] \ln \frac{s^{2}}{s^{2}-4}-g_{3}+2 \\
& +\sum_{\substack{m=2 \\
m \text { even }}}^{\infty}\left(\frac{\tilde{f}_{m}(\lambda)}{(1+\lambda)^{m}}-g_{1}-\frac{2 g_{2}}{m}+\frac{4 g_{3}}{m(m+2)}\right)\left(\frac{2}{s}\right)^{m},
\end{aligned}
$$

where $\tilde{f}_{m}(\lambda)=2^{-m} f_{m}(\lambda)$, and the $g_{i}$ take on the values from (9). Mathematically, (10) is equivalent to (6); numerically, the rate of convergence has improved as the coefficients of $s^{-m}$ now decay faster by a factor of $m^{-2}$. Similarly, for $T_{12}^{Q}$ we have

$$
\begin{aligned}
\frac{(1+\lambda)^{3}}{8} T_{12}^{Q}= & g_{4} \frac{2 s}{s^{2}-4}+\left[g_{5}+g_{6}\left(\frac{1}{4} s^{2}-1\right)\right] \ln \frac{s+2}{s-2}-g_{6} s, \\
& +\sum_{\substack{m=1 \\
m \text { odd }}}^{\infty}\left(\frac{\tilde{f}_{m}(\lambda)}{(1+\lambda)^{m}}-g_{1}-\frac{2 g_{2}}{m}+\frac{4 g_{3}}{m(m+2)}\right) \\
& \times\left(\frac{2}{s}\right)^{m} .
\end{aligned}
$$

Numerical tabulations of $T_{\alpha \beta}^{Q}$ are not given because the expressions and data given above are accurate to at least two significant digits for all $s$. However, we do tabulate the $Q_{\alpha \beta}^{T}$ as they provide a good test of the convergence of the series and may be useful in studies of nearly touching spheres. Expanding (10) about $s=2$ and comparing to (8), we find

$$
\begin{aligned}
Q_{11}^{T}= & -\frac{1}{4} g_{1}-g_{3}+2+\sum_{\substack{m=2 \\
m \text { even }}}^{\infty}\left(\frac{\tilde{f}_{m}(\lambda)}{(1+\lambda)^{m}}-g_{1}-\frac{2 g_{2}}{m}\right. \\
& \left.+\frac{4 g_{3}}{m(m+2)}\right),
\end{aligned}
$$

and similarly

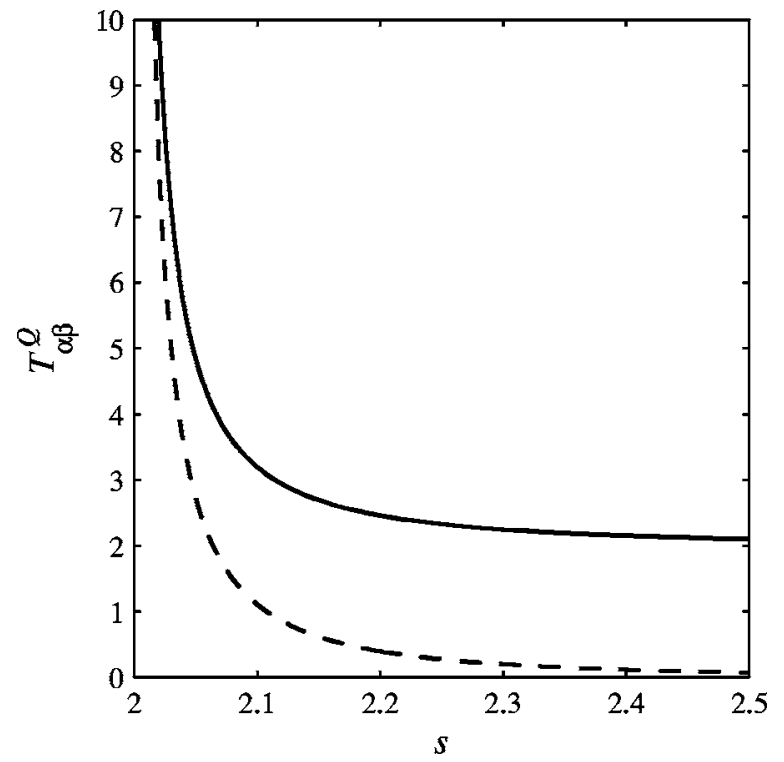

FIG. 1. The functions $T_{11}^{Q}$ (solid line) and $T_{12}^{Q}$ (dashed line) for equal-sized spheres plotted versus the dimensionless separation distance $s$.

$$
\begin{aligned}
\frac{(1+\lambda)^{3}}{8} Q_{12}^{T}= & \frac{1}{4} g_{4}+g_{5} \ln 4-2 g_{6}+\sum_{\substack{m=1 \\
m \text { odd }}}^{\infty}\left(\frac{\tilde{f}_{m}(\lambda)}{(1+\lambda)^{m}}-g_{1}\right. \\
& \left.-\frac{2 g_{2}}{m}+\frac{4 g_{3}}{m(m+2)}\right) .
\end{aligned}
$$

\section{RESULTS FOR $T_{\alpha \beta}^{Q}$}

To illustrate the behavior of the pressure moment, in Fig. 1 we plot $T_{11}^{Q}$ and $T_{12}^{Q}$ as a function of $s$ for identical spheres, $\lambda=1$. Note, the singular behavior of $T_{11}^{Q}$ and $T_{12}^{Q}$ as $s \rightarrow 2$ is proportional to the corresponding force to rate of strain coupling (i.e., to the functions $X_{11}^{P}$ and $X_{12}^{P}$, respectively). In Table I we give results of summing the series (11) and (12) to obtain the $Q_{\alpha \beta}^{T}(\lambda)$. For $\lambda=1$ the series were summed to 300 terms, while for $\lambda \neq 1$ they were summed to 125 termsthe data in Table I are accurate to a minimum of two decimal places (and more where shown). Of course, by summing further terms in the series one can obtain increasingly accurate values of the $Q_{\alpha \beta}^{T}(\lambda)$.

TABLE I. Values of the function $Q_{\alpha \beta}^{T}(\lambda)$, with $\lambda=a_{2} / a_{1}$ the size ratio of the two spheres, appearing in the asymptotic form of $T_{\alpha \beta}^{Q}$ for small separation.

\begin{tabular}{lcccc}
\hline \hline$\lambda$ & $Q_{11}^{T}$ & $Q_{12}^{T}$ & $Q_{21}^{T}$ & $Q_{22}^{T}$ \\
\hline 1 & 2.355 & 0.210 & 0.210 & 2.355 \\
2 & 2.80 & 0.21 & 0.202 & 2.170 \\
3 & 3.20 & 0.19 & 0.179 & 2.120 \\
4 & 3.56 & 0.17 & 0.154 & 2.096 \\
5 & 3.87 & 0.15 & 0.131 & 2.080 \\
10 & 4.96 & 0.08 & 0.064 & 2.043 \\
\hline \hline
\end{tabular}




\section{APPENDIX: LUBRICATION THEORY CALCULATIONS}

Here, we comment briefly on the solution of the Stokes equations in the lubrication approximation used in Sec. III C to calculate the pressure moments of two nearly touching spheres. The flow problem is almost identical to that considered in Sec. 3 of Jeffrey and Corless ${ }^{10}$ - the only difference being that the surface velocity of the deforming sphere (in their notation) is now

$$
\boldsymbol{u}=-\frac{a E}{3}(1+\epsilon-z / a) \boldsymbol{k}+\frac{r E}{3} \boldsymbol{r},
$$

corresponding to an isotropic rate of strain (uniform expansion/contraction) $\boldsymbol{E}=\frac{1}{3} E \boldsymbol{I}$ throughout the sphere. Consequently, in stretched coordinates (choosing the velocity scale $\mathcal{V}=1 / 3 a E)$ the boundary conditions on the deforming sphere are

$$
W=-1-\epsilon(1-Z) \text { and } U=\epsilon R \text {. }
$$

The solution of the lubrication equations proceeds as per Jeffrey and Corless $;{ }^{10}$ full details of the calculations are available upon request.

${ }^{1}$ D. J. Jeffrey, J. F. Morris, and J. F. Brady, "The pressure moments for two rigid spheres in low-Reynolds number flow," Phys. Fluids A 5, 2317 (1993).

${ }^{2}$ Note that the contribution to the force/torque from the trace of the rate of strain can be found from the pressure moments computed by JMB via the symmetry of the grand resistance matrix; the $\boldsymbol{P}$ functions are sufficient.
${ }^{3}$ J. F. Brady, A. S. Khair, and M. Swaroop, "On the bulk viscosity of suspensions," J. Fluid Mech. 554, 109 (2006).

${ }^{4}$ G. K. Batchelor, An Introduction to Fluid Dynamics (Cambridge University Press, Cambridge, 1973).

${ }^{5}$ For a material element in the fluid, $p_{t h}$ is the equilibrium (or thermodynamic) pressure, as computed from the fluid's equation of state, corresponding to the instantaneous density and internal energy of the element. The mechanical pressure, $p_{\text {mech }}$, is defined as the mean normal stress, $p_{\text {mech }} \equiv-\frac{1}{3} \boldsymbol{\sigma}: \boldsymbol{I}=p_{t h}-\boldsymbol{\kappa} \boldsymbol{\nabla} \cdot \boldsymbol{u}$. For an incompressible fluid, $\boldsymbol{\nabla} \cdot \boldsymbol{u}=0$, the mechanical and thermodynamic pressures are equivalent and the former is known to within an arbitrary constant; only the dynamical pressure gradient, $\boldsymbol{\nabla} p$, enters the momentum balance.

${ }^{6}$ Note, we assume that the uniform expansion/compression of the fluid does not lead to macroscopic (i.e., on length scales much larger than the particle size) spatial gradients in the fluid density. Thus, from the continuity equation, the density decreases (increases) exponentially fast in time for a uniform expansion (compression), but it is not a function of spatial position. From the fluid's equation of state, this implies that $p_{t h}$ is, likewise, a function of time only. Hence, one is permitted to take $p_{t h}$ outside the integral for $S^{c}$. Finally, note that the expressions for the pressure moments and formulation of the resistance matrix [see (5)] are valid at any instant in time and are not, therefore, affected by temporal variation of the fluid density and thermodynamic pressure.

${ }^{7}$ D. J. Jeffrey and Y. Onishi, "Calculation of the resistance and mobility functions for two unequal rigid spheres in low-Reynolds-number-flow," J. Fluid Mech. 139, 261 (1984).

${ }^{8}$ D. J. Jeffrey, "The calculation of the low Reynolds number resistance functions for two unequal spheres," Phys. Fluids A 4, 16 (1992).

${ }^{9}$ D. J. Jeffrey, "Higher-order corrections to the axisymmetric interactions of nearly-touching spheres," Phys. Fluids A 1, 1740 (1989).

${ }^{10}$ D. J. Jeffrey and R. M. Corless, "Forces and stresslets for the axisymmetric motion of nearly touching unequal spheres," PCH, PhysicoChem. Hydrodyn. 10, 461 (1988). 